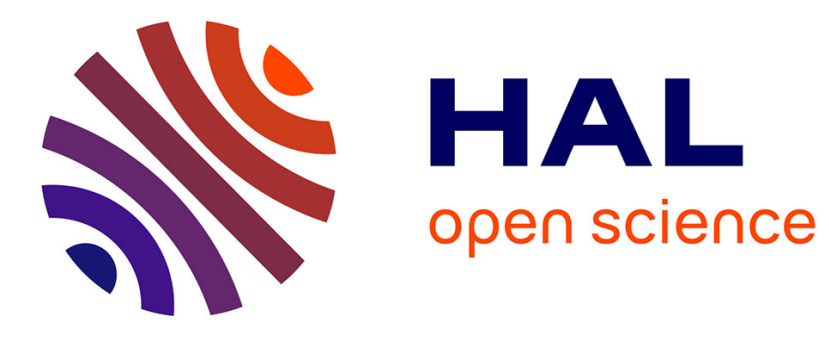

\title{
Closed Peripheral Coverage in Wireless Multimedia Sensor Networks
}

\author{
Amor Lalama, Nesrine Khernane, Ahmed Mostefaoui
}

\section{To cite this version:}

Amor Lalama, Nesrine Khernane, Ahmed Mostefaoui. Closed Peripheral Coverage in Wireless Multimedia Sensor Networks. International Symposium on Mobility Management and Wireless Access, Nov 2017, Miami, FL, United States. hal-02963345

\section{HAL Id: hal-02963345 https://hal.science/hal-02963345}

Submitted on 10 Oct 2020

HAL is a multi-disciplinary open access archive for the deposit and dissemination of scientific research documents, whether they are published or not. The documents may come from teaching and research institutions in France or abroad, or from public or private research centers.
L'archive ouverte pluridisciplinaire HAL, est destinée au dépôt et à la diffusion de documents scientifiques de niveau recherche, publiés ou non, émanant des établissements d'enseignement et de recherche français ou étrangers, des laboratoires publics ou privés. 


\section{Closed Peripheral Coverage in Wireless Multimedia Sensor Networks}

\author{
Amor Lalama \\ FEMTO-ST Institute \\ Bourgogne Franche-Comté University \\ Rue Engel Gros BP 527 \\ Belfort, France \\ amor.lalama@univ-fcomte.fr
}

\author{
Nesrine Khernane \\ FEMTO-ST Institute \\ Bourgogne Franche-Comté University \\ Rue Engel Gros BP 527 \\ Belfort, France \\ nesrine.khernane@univ-fcomte.fr
}

\author{
Ahmed Mostefaoui \\ FEMTO-ST Institute \\ Bourgogne Franche-Comté University \\ Rue Engel Gros BP 527 \\ Belfort, France \\ Ahmed.mostefaoui@univ-fcomte.fr
}

\begin{abstract}
Because of the visual nature (images and videos) of the captured data, the coverage problem in Wireless Multimedia Sensor Networks (WMSNs) is totally different from traditional scalar sensor networks and can be defined in several ways according to the target application. In this paper, we focus on one particular coverage, we called Closed Peripheral Coverage (CPC). This type of coverage is present in several realistic application as military camp surveillance, very sensitive buildings (i.e., nuclear center) surveillance, contaminated region monitoring, etc. The objective hence is to detect any intrusion coming from the outside (viewed by at least one multimedia sensor node). To the best of our knowledge, this is the first research work addressing this issue. Briefly, CPC problem can be defined as follow: after a random deployment of multimedia sensor nodes around the region of interest (i.e., Building to monitor), the goal is to schedule several sets of nodes (i.e., cover sets) such that each of which forms a closed "ring" around the region of interest. Hence, instead activating all nodes at once, the cover sets are scheduled to be activated serially, which ensures a significant network lifetime prolongation improvement. In this paper, we study the CPC problem and present two variants of a centralized algorithm. The preliminary simulation results show clearly the effectiveness of the proposed approaches by a factor varied from 2 to 8 in comparison to the basic approach.
\end{abstract}

\section{KEYWORDS}

Wireless Multimedia Sensor Networks; Coverage Problem; Closed Peripheral Coverage (CPC)

\section{ACM Reference format:}

Amor Lalama, Nesrine Khernane, and Ahmed Mostefaoui. 2017. Closed Peripheral Coverage in Wireless Multimedia Sensor Networks. In Proceedings of MobiWac'17, Miami, FL,USA, November 21-25, 2017, 8 pages. https://doi.org/10.1145/3132062.3132073

Permission to make digital or hard copies of all or part of this work for personal or classroom use is granted without fee provided that copies are not made or distributed for profit or commercial advantage and that copies bear this notice and the full citation on the first page. Copyrights for components of this work owned by others than ACM must be honored. Abstracting with credit is permitted. To copy otherwise, or republish, to post on servers or to redistribute to lists, requires prior specific permission and/or a fee. Request permissions from permissions@acm.org.

MobiWac'17, November 21-25, 2017, Miami, FL,USA

() 2017 Association for Computing Machinery.

ACM ISBN 978-1-4503-5163-8/17/11 .. \$15.00

https://doi.org/10.1145/3132062.3132073

\section{INTRODUCTION}

Advances in multimedia hardware and wireless communication technologies have allowed the emergence of Wireless Multimedia Sensor Network (WMSNs) with small size, low-cost, and multifunctional sensors equipped with multimedia sensing unit. WMSNs have several important applications in various domains that require not only the intrusion detection but also the identification, the tracking and the surveillance of target objects such as: critical resource protection, military camp, airport, prisons and country borders. Thus, constructing a Multimedia Sensors Coverage (MSC) is an efficient way for such applications since they can provide more detailed information (i.e. real vision) of the Region Of Interest (ROI). At the difference with traditional wireless sensor networks, where the sensing region of the sensor node is represented by a disk model [3], in the WMSNs, the multimedia sensors nodes may have a limited sensing region represented by a Field of View (FoV), usually represented as a cone. Due to this difference, coverage algorithms for traditional sensor networks can not be applied straightforwardly and efficiently in WMSNs

We distinguish three main types of coverage in WMSNs $[1,4,16]$ : (a) area coverage, (b) target coverage and (c) barrier coverage. The area coverage refers to monitoring the whole considered area and any change within the later should be detected immediately (e.g. Volcano area, Fire detection). While the Target coverage allows the coverage of one or more objects in a well-defined area without the need to monitor the whole area (e.g. monitoring of a historic monument in an exhibition instead of the entry hall). Finally the barrier coverage where there is no need to cover the whole region, but only construct a line of Multimedia sensor networks and thus to detect the crossing objects (e.g. barrier deployment on the roadway to detect the wild animals crossing). Figure 1 illustrates an example of these three different coverage types in WMSN.

In this paper, we introduce and study a new type of coverage related to WMSNs, named Closed Peripheral Coverage (CPC). The motivation behind this type of coverage comes primarily from realistic applications and scenarios. In fact, we present hereafter, not in an exclusive way, some illustrative applications where CPC is required.

- Military camp surveillance: in mobile deployment of military troops, especially in hostile environment, it is often needed to know, as earlier as possible, any intrusion coming from the outside what ever the direction is. In addition, when the deployment is large, it is usually very hard to mobilize as many soldiers as necessary for this purpose. Here, using WMSNs specifically dedicated to form a surveillance 


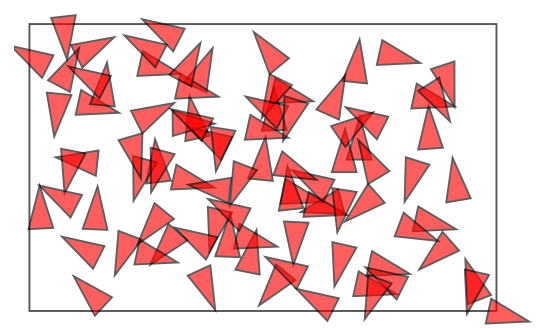

(a)

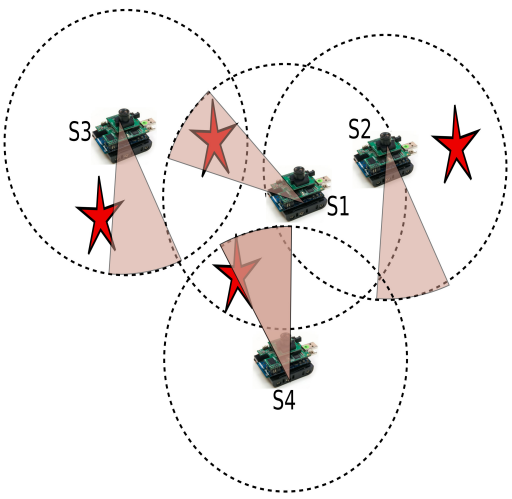

(b)

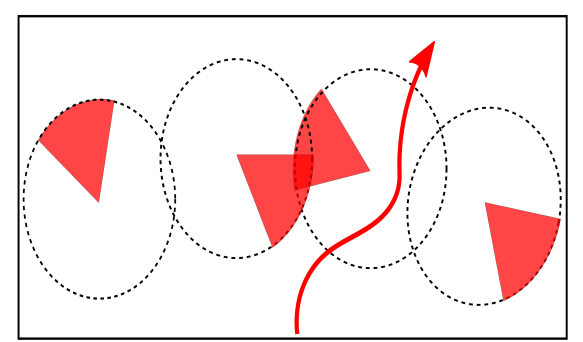

(c)

Figure 1: Different types of coverage in WMSN: (a) Area Coverage; (b) Target Coverage; (c) Barrier Coverage and (d) Peripheral Coverage.

ring around the camp and trigger alarms as quickly as intrusions are detected will be very useful.

- Sensitive region monitoring: this scenario was inspired from a real event happened in the south desert of Algeria in January 2013, where a gas facility located in Tigentourine was attacked by a terrorist group from Al-Qaida [17]. The attack led to more than 60 people killing. As the gas facility was located in large and somehow isolated region, the "local" monitoring, formed from conventional video surveillance cameras, was not efficient to early alert the army forces about the planned attack. We believe that a large deployment of WMSNs sufficiently far around the gas facility could provide valuable information sufficiently in advance to military forces in order to prevent this type of attacks.

- Contamination Monitoring: let us consider a scenario in which a region was contaminated by an epidemic disease. In this case, the health authorities may decree a confinement measurement in order to stop the propagation of the contamination. A deployment of WMSNs may help in implementing such a confinement by alerting about any violation of the latter.

All the above mentioned applications and scenarios, as many others, need the deployment of WMSNs with closed peripheral coverage requirement.

Our contribution. Although several researchers have proposed coverage solution algorithms within the context of WMSNs, none has, to the best of our knowledge, considered the CPC problem in WMSNs (we used sometimes the word camera to design the multimedia sensor node). In summary the research problem we are facing is: given a set of randomly deployed multimedia sensors, we are looking for an algorithm that is able to form a set of closed peripheral covering sets where each of which forms a closed ring; i.e., any moving object towards the region of interest will cross at least the field of view (FoV) of at least one the sensor within the activated set.

Identifying and forming such covering sets, with of course minimal cardinality is a challenging research issue, in particular with very limited resources multimedia sensors. To this end, we propose, in this paper, an algorithm that ensures a closed peripheral coverage with two variants. We conducted several simulation studies in order to measure the effectiveness of the proposed approaches. The obtained results show clearly that our approaches improves over the basic approach by a factor varying from 2 to 8 .

Roadmap. The reminder of this paper is organized as follows: Section 2, presents the related work. In section 3, we present the preliminaries and the main definitions used in this paper. While, in section 4 , we give a definition of closed peripheral coverage problem. In Section 5, we will explain the peripheral coverage test and we will present the proposed two variants for our Centralized Closed Peripheral Coverage Algorithm (CCPCA) to resolve the latter problem. The simulation results of CCPCA algorithm are presented in section 6 and section 7 concludes our paper.

\section{RELATED WORK}

Wireless Multimedia Sensor Network is a promising technology for various applications, and it will be increasingly necessary to use for monitoring applications. However, WMSNs have inherent coverage problem due to the limited resources of video sensors (taking into account both: scalar and multimedia data) compared to the application requirements. To this end, there has been rich literature. We briefly introduce some work.

To the best of our knowledge the barrier coverage problem in wireless camera sensor networks was first presented in [13], in which the solution was divided into three phases. The first one consists in collecting the geographical information of neighboring nodes in order to determine the barrier lines. Then in the second phase, a Barrier Request (BREQ) packet was used to determine the candidate barrier lines. Finally, the third phase used a Barrier Reply (BREP) packet to take a decision about the efficient barrier line in a wireless camera sensor network.

The primary focus of [10] is to minimize the number of camera sensors in the full-view barrier coverage. To solve the problem, the target field was partitioned into two regions: a) Full-View-Covered 
regions and b) Non-Full-View-Covered regions. In the Full-ViewCovered regions a weighted directed graph was considered in order to define a minimum source-destination path. Based on the minimum source-destination path solution, an optimal algorithm for minimum camera barrier coverage was proposed.

Different from the aforementioned researches, authors in [18] addressed the concept of strong camera barrier problem with kcoverage problem for $k<=2$. To solve the problem, the authors, firstly, formulate the problem as an Integer Linear Programming (ILP) based on a barrier coverage model. Then, based on the clustering architecture a barrier coverage mechanism was proposed, where each cluster header constructs the defence curve. Finally, to evaluate the effectiveness of the proposed solution, an extensive simulation was provided.

To deal with the problem of k-coverage, authors in [15] have proposed a scheme for establishing $\mathrm{k}$-barrier coverage within a long belt-like region using a randomly deployed directional sensor nodes. The proposed scheme considers both, the location and the antenna direction of nodes. To construct k-barrier in a distributed manner, four phases have been detailed: initial starting node selection phase, remaining starting nodes selection phase, active node selection phase and roll back phase. The simulation results have shown that the directional sensing scheme outperforms the omni-directional model in terms of success rate and the number of active sensors.

However, all of the aforementioned researches consider the coverage of only one side of the target. Thus, these solutions are invalid in some applications, especially, when the target requires a peripheral coverage such a military camp, in which a small flaw can entail big damages.

To overcome with a peripheral coverage, a lot of solutions were proposed for traditional sensor networks. In [8], authors addressed the coverage of a large target object. The proposed solution intends to minimize the amount of energy by identifying the minimum cover set in a distributed way. Three phases was presented: finding the minimum cover, greedy forward neighbour and finding multiple minimum covers. Then, a formal proof of correctness and convergence time analysis of the proposed algorithm was provided.

Authors in [5] have proposed a solution to deal with both, border and area coverage. To build a border coverage, authors used minimum dominating sets and the concept of Located Voronoi Polygons (LVP) (i.e. graph theory concept). The proposed solution runs through three phases: first one consists of identifying the nodes close to the border (i.e. boundaries nodes). The second phase is used to identify whether the nodes are transmitter or internal nodes. Finally, the last phase concerns the failure cases which was resolved by replacing border nodes by the transfer nodes. Simulation results show that the proposed protocol achieves a high coverage ratio in comparison to some known protocols

However, these solutions can not be reported in wireless multimedia sensor networks and this for two crucial reasons. Firstly, in traditional sensor networks, sensing region is represented by a disc, while in WMSN, the sensing region is limited and represented by an Field of view ( $F o V)$. Therefore, the proposed solution should take into account not only the node deployment of multimedia sensor nodes but also the direction of the latter. Secondly, in traditional sensor networks the sensing and encoding process is characterized

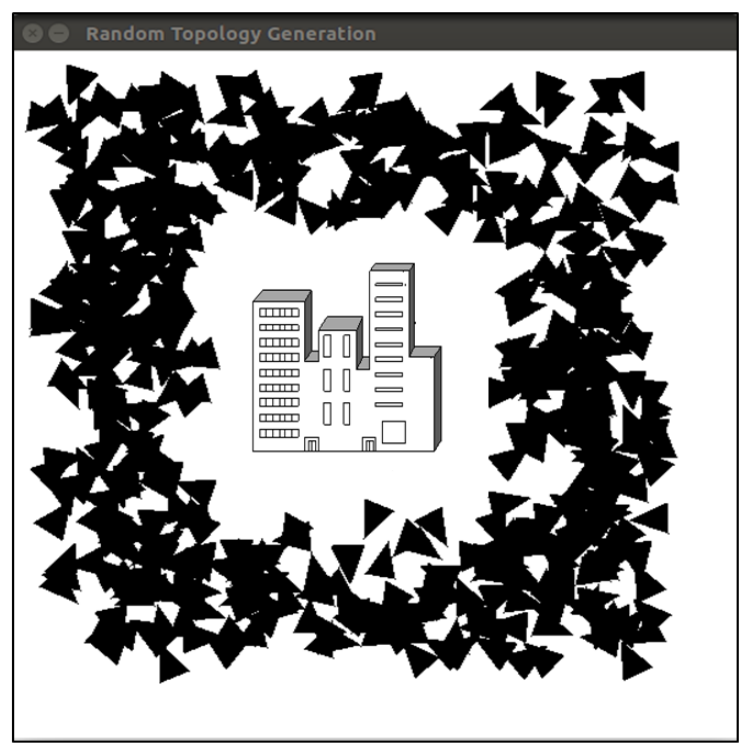

Figure 2: Example of random deployment of multimedia node in two-dimensional rectangle.

by a low power consumption, while in WMSN, the encoding process requires much more resources due to the multimedia content. Accordingly, the problem of finding the minimum cover should be addressed in WMSN to ensure a maximum network lifetime.

\section{BACKGROUND AND DEFINITIONS}

To formulate the problem of peripheral coverage treated in this paper, in this section, we present network model, the coverage model of multimedia sensor node. Then define several terminologies and notations that will be used to formulate our problem.

\subsection{Preliminaries}

We assume that our Region of Interest (ROI) is a two-dimensional rectangle of size $L \times W$, where $L$ and $W$ represent the length and the width of the monitoring region $(R O I)$, respectively. We also assume a network of $N$ multimedia sensor nodes randomly deployed with a uniform manner around monitoring building or area. The multimedia sensor nodes are static (no mobility consideration) and the cameras are fixed (not rotative). Figure 2 illustrates an example of random deployment of multimedia sensor networks studied in this paper.

Let us recall that, the multimedia sensor nodes have a limited sensing region represented by a Field of View (FoV), from which, an adopted sector is used to represent the coverage model of multimedia sensor node. We use $v_{i}$ to denote a multimedia sensor node $i$, Each multimedia node $i$ is represented by the 5-tuples $v_{i}\left(x_{i}, y_{i}, A o V, D o V, \beta, R_{c}\right)$, where $x_{i}, y_{i}$ represent the location Cartesian coordinates of the node $i, A o V$ and $D o V$ represent the angle of view and depth of view respectively. $\beta$ is the orientation angle and $R_{c}$ is the communication range. All the deployed multimedia sensor nodes are functionally equivalent (i.e. the same $\mathrm{AoV}, \mathrm{DoV}$ 
and $R_{c}$ ), and they are able to obtain their location information by a built-in GPS or using some localization algorithms $[2,7,12]$

\subsection{Definitions}

3.2.1 Field of View (FoV). As mentioned before, we adopt a sector as a model of coverage for multimedia sensors; this sector is called fields of view. The first definition of Field of View (FoV) was given in [6], where it is defined as the maximum volume (in $3 \mathrm{D})$ or maximum area (in 2D) visible from a camera, that can be determined by: (a) node location coordinate, (b) Angle of View $(\mathrm{AoV}),(\mathrm{c})$ Depth of View $(\mathrm{DoV})$ and (d)the orientation angle.

3.2.2 Angle of View (AoV). The angle between the left and the right limits of the FoV is defined as the AoV. In figure 3, the AoV is delimited by the three segment $[v d],[d g]$ and $[g v]$ and denoted by $\alpha$.

3.2.3 Depth of View (DoV). The farthest distance that the multimedia sensor node can identify an object represents the Depth of View [6].

3.2.4 Communication range $\left(R_{c}\right)$. The communication range represent the farthest distance through which the multimedia sensor node $i$ can communicate with another multimedia node $j$ [6]. Figure 3 shows a graphical presentation of FoV, DoV, AoV and $R_{c}$.

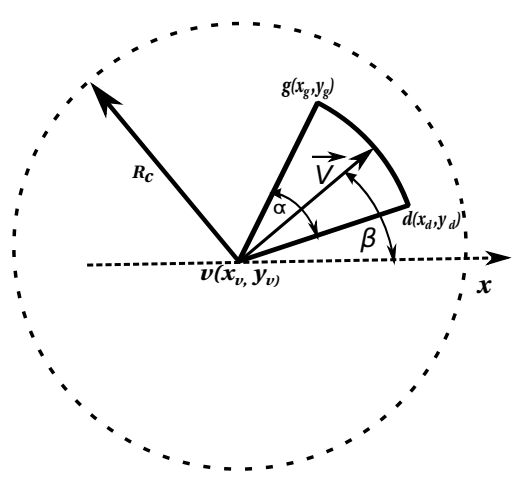

Figure 3: Coverage model of multimedia node.

We say that a two-dimensional point $p_{j}(x j, y j)$ is covered by a multimedia sensor node $v_{i}\left(x_{i}, y_{i}, A o V, D o V, \beta, R_{c}\right)$ if and only if $p_{j}$ is within the $\boldsymbol{F o} \boldsymbol{V}$ of the node $v_{i}$. This condition can be interpreted as the following:

(1) $\operatorname{dist}\left(v_{i}, p_{j}\right) \leq \operatorname{DoV}$ where $\operatorname{dist}\left(v_{i}, p_{j}\right)$ represents the Euclidean distance between node $v_{i}$ and the point $p j$.

(2) The angle between the two vector $\vec{V}$ and $\overrightarrow{v_{i} p}$ is in the interval $[\beta, \beta+A o V]$.

The following method can be used to verify if the node $v_{i}$ covers the point $p:$ if $\left\|\overrightarrow{v_{i} p_{j}}\right\| \leq D o V$ and $\overrightarrow{v_{i} p_{j}} \cdot \vec{V} \geq\left\|\overrightarrow{v_{i} p_{j}}\right\| \cos (A o V)$, then $p_{j}$ is covered by $v_{i}$.

Figure 4 shows an example of a point $p_{j}$ covered by a multimedia sensor node $v_{i}$. From the previous definitions, we can say that an area $A$ is covered by a multimedia sensor node if and only if all points of this area are inside the $\mathrm{FoV}$ of this node.

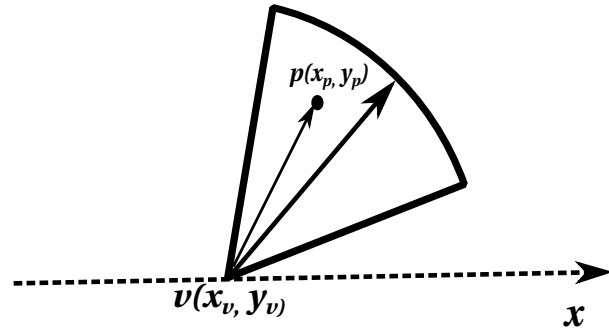

Figure 4: Multimedia sensor node $v_{i}$ cover the point $p_{j}$.

\section{CLOSED PERIPHERAL COVERAGE PROBLEM}

After providing the network model and definitions, in this section, we will examine the $\mathrm{CPC}$ problem. To illustrate the latter, we consider the example of figure 2 , where a set of $N$ multimedia sensor nodes are deployed around a sensitive building(e.g., temporary military camp, zoo or a nuclear central,...etc.). The main objective, here, is to detect, as early as possible, any intrusion coming from the building periphery to the centre or evasion of the sensitive area. Usually, a hostile or insecure situations where the impossibility of human intervention are considered for the utilization of this kind of network. Thus, the random deployment by air-plane or other methods have become necessary in this case. Note that in a random deployment, generally, the density of nodes must be sufficient to ensure the requirement of coverage quality in critical applications which causes a nodes redundancy. In this case, and taking into account the limited resources of sensor nodes (energy, processing, cost...,etc), it is not necessary to activate all nodes in ROI. The best solution is to divide the initial deployed nodes set into a disjoint subsets ensuring a closed peripheral coverage. Every subset $S_{i}$ can achieve the peripheral coverage for $t_{i}$ time which increases sufficiently the network lifetime in contrast with the activation of all deployed nodes. Figure 5 illustrates a real example of a random deployment of multimedia sensor nodes and three disjoint peripheral coverage subsets resulting from the initial deployment. This problem raises two issues: (a) how can we decide if the deployed nodes form a peripheral coverage for the monitoring building before starting the disjoint subsets computation?, (b) how can we calculate the maximum disjoint subsets of multimedia sensors nodes which assuring the peripheral coverage?

\section{CLOSED PERIPHERAL COVERAGE ALGORITHM}

In this section, we present our proposed solution for the closed peripheral coverage in multimedia sensor networks. The latter is a centralized algorithm named Centralized Closed Peripheral Coverage Algorithm (CCPCA). This algorithm is divided into two variants: Spiral Periphery Outer Coverage (SPOC) and Spiral Periphery Inner Coverage (SPIC). We will explain the both variants later. Based on the information sent from the deployed nodes in the ROI, the set of disjoint subsets of multimedia sensor nodes to be activated is calculated at the sink level. After obtaining the set of disjoint subsets, the sink sends an activation directive to the 


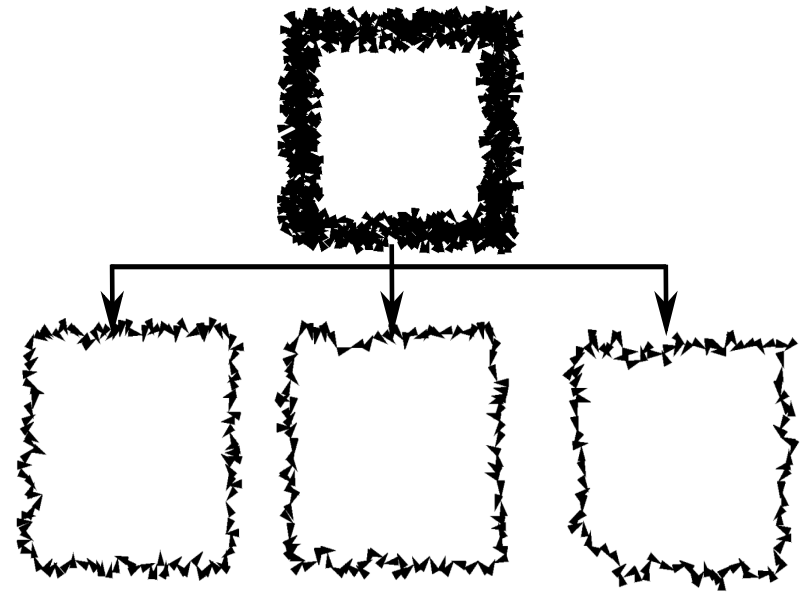

Figure 5: The initial deployment and the three Closed peripheral coverage subsets.

concerned nodes (namely, nodes of the first subset) to proceed the monitoring of the ROI. Note that the activation of the $i^{t h}$ subset is done after the failure of the $i^{t h}-1$ subset.

\subsection{Peripheral coverage test}

Let us recall that the main objective of this paper is to ensure a closed peripheral coverage. Thus, finding a method to test the existence or not of the peripheral coverage is crucial task before starting the computation of the closed peripheral coverage sets. To this end, we proceed by separating the region into three subregions: Outside Network Region (ONR), Inside Network Region (INR) and Network Coverage Region (NCR). In the following, we will describe these subregions.

5.1.1 Network Coverage Region (NCR). We define the NCR as all points covered by the deployed multimedia sensor nodes in the ROI. In figure 6, the set of black triangles represents the NCR region.

5.1.2 Outside Network Region (ONR). The ONR region is defined as the set of points which located in the outside of the NCR region. As shown in figure 6, the blue color represents the ONR region.

5.1.3 Inside Network Region (INR). The INR region is the set of points situated between the NCR and the centre of ROI. In figure 6, the green color represents the INR region.

Given $V$ as a set of Multimedia sensor nodes, We say that the set of Peripheral Coverage Network $P C N \subset V$ is forming a Closed Peripheral Coverage if and only if there is no overlapping between the two region ONR region and INR region.

This condition can be formulated as following: $O N R \cap I N R=\Phi$.

\subsection{Centralized Closed Peripheral Coverage Algorithm (CCPCA)}

After the deployment of multimedia sensor nodes in the ROI, each node reports its information (namely. $x_{i}, y_{i}, A o V, D o V, \beta, R_{c}$ ) to the sink using a geographic routing protocols proposed in $[11,14]$.

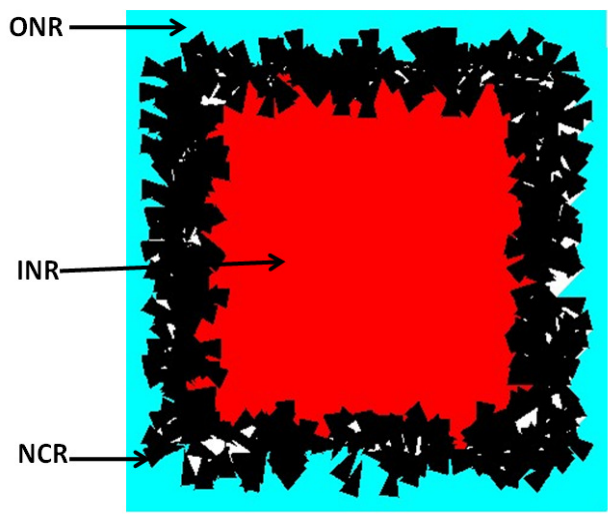

Figure 6: The NCR, ONR and INR regions.

Based on the collected information from the deployed multimedia sensor nodes in the ROI, the sink executes the CCPCA algorithm to compute the possible peripheral coverage subsets. Two scenarios will be studied and defined in the following.

5.2.1 Scenario 1: Without routing phase influence. In this first scenario, we consider two cases. In the first one, the network is composed only of the multimedia sensor nodes. We assume that the energy consumed by the multimedia sensor nodes in processing phase (intruder identification, compressing..., etc) is very higher than the energy consumed in the information transmission phase. As an example of this case, a monitoring application where the role of the multimedia sensor nodes is informing the sink of any intruder presence by sending only its type information. In this case, the computation of peripheral coverage sets is not influenced by the routing phase. The execution of the latter scenario is shown in Algorithm 1. The sink selects gradually at each stage the nearest (respectively the farthest) multimedia sensor node to the ROI centre for SPOC variant (respectively for SPIC variant). If the deletion of this node doesn't cause an overlapping between ONR and INR regions (i.e. No violation of coverage test), the sink suspend this node from the list of initial deployed multimedia sensor nodes (line 12,13 and 14 in algorithm 1). Otherwise, the sink add this node to the $i^{\text {th }}$ Peripheral Coverage $P C_{i}$ (line 14 and 15). The resulting cover set $P C_{i}$ will be excluded from the set $V$ in order to calculate the $P C_{i+1}$ (line 18). The sink repeats the previous steps until violation of non-overlapping condition.

In the second case, we suppose that the role of the multimedia nodes is the data gathering from the ROI. Whereas, the scalar sensor nodes are responsible of the information routing from the multimedia sensor nodes to the sink $[11,14]$. To calculate the peripheral coverage sets, the sink uses the algorithm 1 . Then it calculates the conventional sensor node set which will participate for the data routing phase. To calculate the $i^{t h}$ Peripheral coverage set, the sink removes the $i^{t h}-1$ peripheral coverage set from the set $V_{i}$. Note that, the sink calculates a disjoint routing set of scalar sensor nodes for each peripheral coverage set. in other words, a newest disjoint routing set for each peripheral coverage set. 


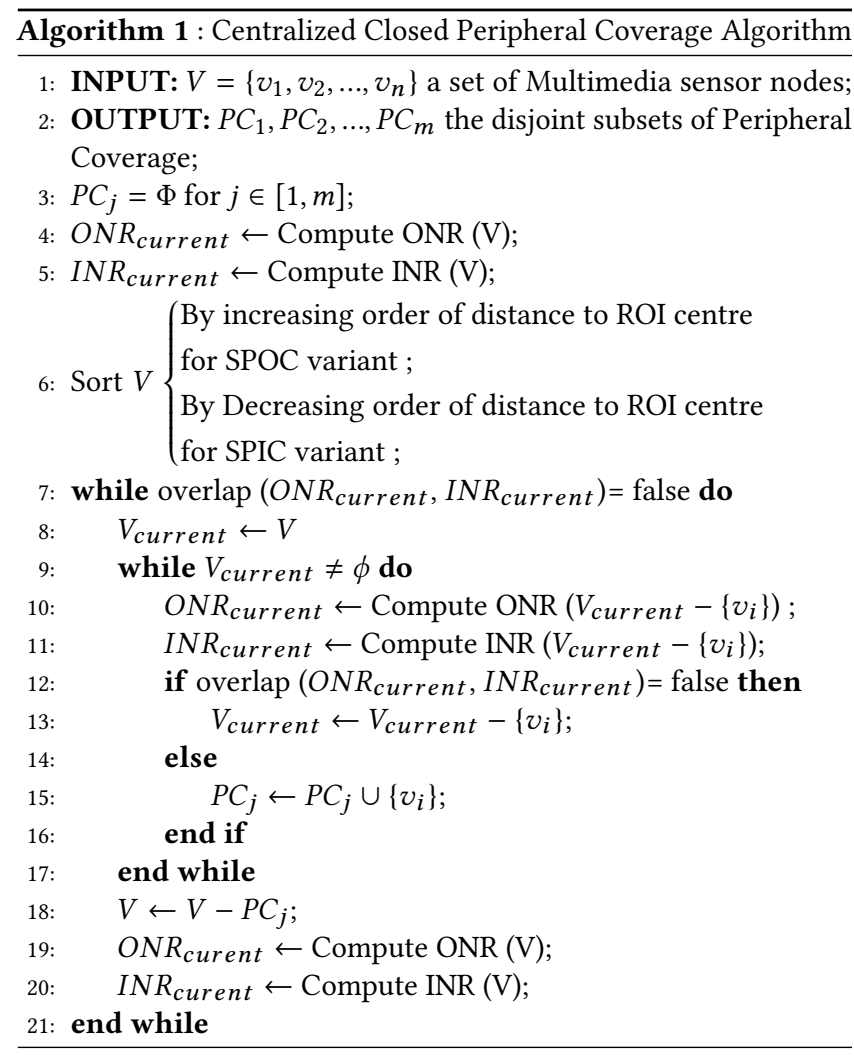

5.2.2 Scenario 2: Routing phase with multimedia sensor nodes. In this scenario, we assume that the multimedia sensor nodes are responsible for both data gathering and data delivering to the sink In this case and owing to high energy consumption for the transmission phase, in addition to the disjoint peripheral coverage sets, the sink calculates a disjoint routing set for each peripheral coverage set. So that to illuminate the influence of transmission phase in the computation of peripheral coverage set. For example, after the computation of $i^{t h}$ peripheral coverage set, the sink calculates also the corresponding $i^{t h}$ routing set. To compute the $i^{t h}+1$ peripheral coverage set, the sink removes the $i^{t h}$ peripheral coverage set and ${ }_{i}{ }^{t h}$ routing set from the set $V_{i}$.

\section{SIMULATION SETTINGS AND PERFORMANCE RESULTS}

In this section, our proposed algorithm will be evaluated through comparing the SPOC and SPIC variants by implementation in $\mathrm{C}++$ using the SDL library [9]. Let us recall that the main condition to ensure a peripheral coverage is that the $O N R \cap I N R=\Phi$. Therefore, we should, firstly, determine the required number of multimedia sensor nodes to ensure at least one peripheral coverage set. To this end, we assess the impact of network density on peripheral coverage success rate taking into account different values of area size and multimedia sensor nodes parameters (i.e AoV and DoV). Secondly, and according to the two scenarios cited in the section 5.2, we study the impact of number of nodes on the number of disjoint peripheral cover sets by fixing the area size and nodes parameters at

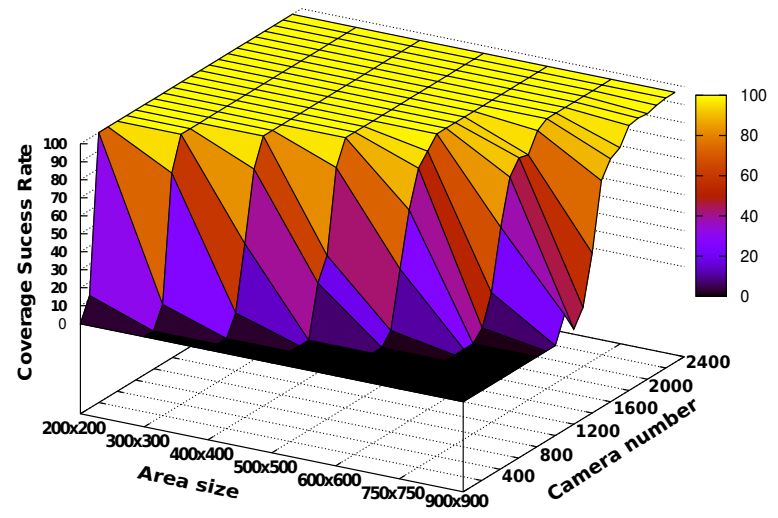

Figure 7: 5: Peripheral Coverage Success Rate Vs Camera number in different area sizes.

some values. In addition of these metrics, we evaluate the minimum number of sensor nodes required to ensure the data delivery from each multimedia sensor node to the sink. For the second scenario, we evaluate the impact of the desired peripheral coverage sets number on the number of multimedia sensor nodes required, while ensuring a disjoint routing set for each peripheral coverage set.

\subsection{Settings}

The CCPCA Algorithm requires prior existence of peripheral coverage to start the computation process of the disjoint peripheral coverage subsets. For this raison, we study the impact of area size and network density on the minimum of cameras number required to ensure, at least, one peripheral coverage set. Then, the computation of the disjoint peripheral coverage sets can be affected.

Each simulation point is executed 500 times to reduce the effect of the random topology. The coverage success rate is calculated by dividing the number of achieved peripheral coverage by the number of simulations (i.e. 500 here) which we can consider it as a probability of coverage success.

To assess the minimum number of camera sensor nodes required to ensure a peripheral coverage, we study the impact of camera number on the peripheral coverage success rate with different area size. The latter is varied from, $200 m \times 200 m$ to $900 m \times 900 m$. The cameras sensors are randomly and uniformly deployed in the Region of Interest with the usual multimedia sensor node parameters: $\mathrm{AoV}=\pi / 6$ and $\mathrm{DoV}=30 \mathrm{~m}$. Figure 7 shows a proportional relationship between the peripheral coverage success rate and the number of cameras sensors in different area size. From the latter figure, we can observe that, more is the number of camera nodes (deployed in the ROI), higher is the success rate for forming a peripheral coverage. On the other hand, we can observe that the area size and the peripheral coverage success rate are inversely proportional. That means, when the area size increases, the number of camera sensor nodes required to ensure a $100 \%$ of the coverage success rate increase also. For any area size and based on previous results, we can determine the minimum number of camera sensor nodes to deploy in order to ensure a peripheral coverage. Taken an example from the figure 7, for the following area size: $200 \times 200,500 \times 500$ and $900 \times 900$, area size, in which 200,800 and 2300 camera sensor 


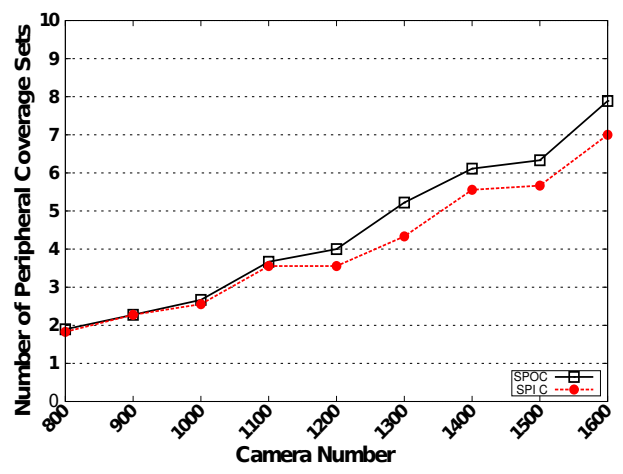

Figure 8: Number of peripheral coverage sets in SPOC and SPIC variants.

nodes were, respectively, required to ensure a peripheral coverage with $100 \%$ of success rate.

\subsection{Number of Peripheral Coverage set}

In this section, we will evaluate our proposed algorithm over the two scenarios cited in 5.2 where we compare the two variants, SPOC and SPIC.

6.2.1 Scenario 1: Without routing phase influence. In this scenario, we suppose two cases. In the first case, we suppose that only the multimedia sensor nodes are deployed in ROI to ensure the peripheral monitoring task of the sensitive building or dangerous area. In the monitoring task, The objective of is to identify the intruder and send as soon as possible the type of intruder to the sink to start the appropriate operation. Therefore, we suppose that the energy consumed for the data transmission is negligible. In the second one, we suppose that there is an hybrid deployment of nodes. The multimedia sensor nodes gather the information from the ROI and the scalar sensor nodes issue the gathered data from the multimedia sensor nodes to the sink. For the both cases, there isn't an influence of the data routing phase on the peripheral coverage set computation.

In first case, we study the relationship between the number of peripheral coverage and the number of multimedia sensor nodes. We compare the simulation results of the both variant, SPOC and SPIC. With the usual multimedia sensor nodes parameters in an area of $500 \mathrm{~m} \times 500$, we variate the number of camera sensors from 800 to 1600 and we observe the peripheral coverage sets result. Each simulation result is represented by the means of 30 times executions. Figure 8 illustrates the simulation results. In both variants SPIC and SPOC, we can observe a proportional relationship between the cameras number deployed in the ROI and the Number of peripheral coverage sets. This result can be explained by the fact that, more the deployed camera number in ROI, more the area covered which increase the peripheral coverage sets result. As shown in the figure 8, for 800 node deployed in ROI, the obtained peripheral cover sets is equal to 2 in SPOC and SPIC. While, for 1600 nodes, we obtain 7 and 8 peripheral coverage sets for SPOc and SPIc, respectively. Even thought, there is a slight difference between the SPOC and SPIC results. However, the SPOC provides better results than the SPIC. We can explain this difference by the fact that SPOC variant starts

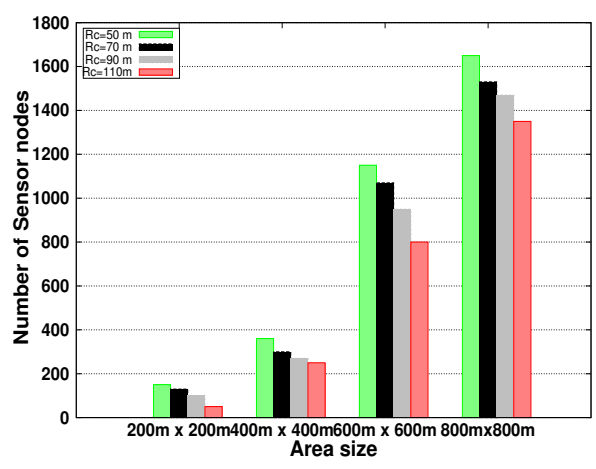

Figure 9: The number of scalar sensor required to ensure the routing phase for 1 peripheral coverage set with $R_{c}$ variable in different area sizes.

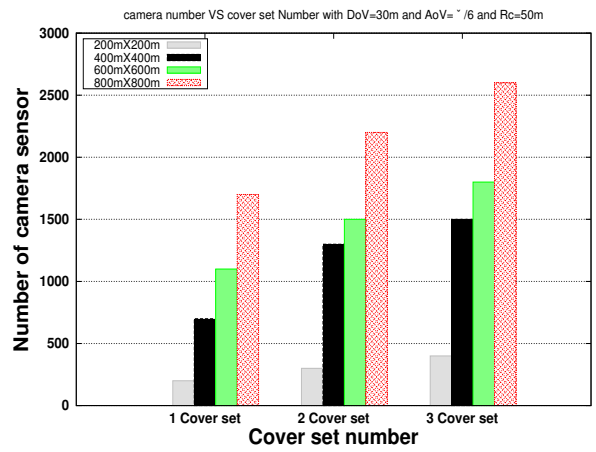

Figure 10: The number of camera sensor nodes required VS number of peripheral coverage set in different area sizes.

the peripheral coverage set computation from the outside, which allows to use the more relevant camera sensor nodes to calculate the peripheral coverage sets.

In the second case, we consider that only the scalar sensor nodes are used to route the data gathered by the camera sensor nodes. Based in this purpose, we study the impact of the area size and the communication Range $\left(R_{c}\right)$ on the number of scalar sensor nodes required to ensure the data delivering from the camera sensor node to the sink. Figure 9 shows how the number of sensor nodes needed to ensure the routing phase increase when the area size increase. In an other hand, when the $R_{c}$ increases, the number of sensor node decrease. So to ensure one peripheral coverage set in area of $400 \times 400$ with $R_{c}=70 \mathrm{~m}$, we must deploy at least 300 scalar sensor nodes but for the same $R_{c}$, we need more than 1000 scalar sensor nodes in area of $600 \mathrm{~m} \times 600 \mathrm{~m}$.

6.2.2 Scenario 2: information routing with the camera sensor nodes. In this second scenario, the camera sensor nodes take the both operations: the data gathering and the information routing. In this case the calculation of the peripheral coverage set can be influenced by the information routing phase due to the high energy consumption by the camera sensor nodes in this phase. For this raison, we evaluated the number of camera sensor nodes required to ensure 1, 2 and 3 peripheral coverage set in different area size 
with $\mathrm{AoV}=\pi / 6, D o V=30 \mathrm{~m}$ and $R_{c}=50 \mathrm{~m}$. Figure 10 shows that if there are more required peripheral coverage sets, the number of camera sensor nodes to ensure this objective is higher. In the same sense, more the area is large; more the required number of camera sensor nodes is higher.

\section{CONCLUSIONS}

In this paper, we propose a centralized Algorithm, CCPCA, to calculate the closed peripheral coverage in Multimedia Wireless Sensor Network. To the best of our knowledge, this paper is the first work that treats the closed peripheral coverage problem in MWSN. This algorithm divided the deployed camera sensor nodes on disjoint subsets in order to increase the network lifetime. Then, was solved over two variants: (a) Spiral Periphery out coverage (SPOC) and (b) Spiral Periphery Inner Coverage (SPIC). The first variant started by forming the outer peripheral coverage to the center of ROI. While, the second variant started by forming the inner peripheral coverage set to the center of Our ROI. Our algorithm was evaluated through simulations where we have considered two different scenarios. The first one presents the case where the information routing phase has no impact on the calculation of peripheral coverage subsets. While, in the second one, only the multimedia sensor nodes are deployed in the monitored area, and have to ensure both: data collect and data routing. The simulation results showed clearly that we can increase considerably the network lifetime from 2 to 8 times in comparison to the basic approach. This result was obtained by dividing the deployed camera sensor nodes on disjoint subsets. In other hand, we observe a slight different between SPOC and SPIC. The SPOC variant had given a better number of peripheral coverage set than the SPIC variant. This difference can be explain by the fact that SPOC variant engaged more multimedia nodes in computation of peripheral coverage sets because it started by forming the outer set progressively. The latter subsets are activated in scheduled manner. By analysing the obtained results, we have observed the important redundant number of cameras nodes that are unused during the peripheral coverage calculation and caused by the random deployment and the limited field of view. For this raison, in our future work we will focus on exploration of the cameras parameters (namely. Rotation, mobility and Zoom) to involve the unused node on the peripheral coverage computation, while ensuring a distributed control.

\section{REFERENCES}

[1] Ian F Akyildiz, Tommaso Melodia, and Kaushik R Chowdhury. 2007. A survey on wireless multimedia sensor networks. Computer networks 51, 4 (2007), 921-960.

[2] Nirupama Bulusu, John Heidemann, and Deborah Estrin. 2000. GPS-less low-cost outdoor localization for very small devices. IEEE personal communications 7, 5 (2000), 28-34.

[3] Chih-Yung Chang, Chih-Yao Hsiao, and Yu-Ting Chin. 2014. The k-Barrier Coverage Mechanism in Wireless Mobile Sensor Networks. In Proceedings of the 12th International Conference on Advances in Mobile Computing and Multimedia. ACM, 275-280.

[4] Chien-Fu Cheng and Kuo-Tang Tsai. 2012. Distributed Barrier Coverage in Wireless Visual Sensor Networks With $\beta$-QoM. IEEE Sensors fournal 12, 6 (2012), $1726-1735$.

[5] Ali Dabba and Rachid Beghdad. 2014. BCP: A Border Coverage Protocol for wireless sensor networks. In Science and Information Conference (SAI), 2014. IEEE, 632-640.

[6] Ugur Murat Erdem and Stan Sclaroff. Optimal placement of cameras in floorplans to satisfy task requirements and cost constraints. In Omnivis2004, The fifth Workshop on Omnidirectional Vision, Camera Networks and Non-classical cameras. Citeseer.

[7] Aram Galstyan, Bhaskar Krishnamachari, Kristina Lerman, and Sundeep Pattem. 2004. Distributed online localization in sensor networks using a moving target. In Information Processing in Sensor Networks, 2004. IPSN 2004. Third International Symposium on. IEEE, 61-70.

[8] Ka-Shun Hung and King-Shan Lui. 2010. On perimeter coverage in wireless sensor networks. IEEE Transactions on Wireless Communications 9, 7 (2010), 2156-2164.

[9] N Kikuchi, Y Shigeta, K Miyake, W Tanaka, and M Nabeta. 1989. An Integrated System Development Method and Support System based on SDL and C++, SDLâĂŽ89: The Language at Work. (1989).

[10] Huan Ma, Meng Yang, Deying Li, Yi Hong, and Wenping Chen. 2012. Minimum camera barrier coverage in wireless camera sensor networks. In INFOCOM, 2012 Proceedings IEEE. IEEE, 217-225.

[11] Samir Medjiah, Toufik Ahmed, and Francine Krief. 2010. AGEM: adaptive greedycompass energy-aware multipath routing protocol for WMSNs. In Consumer Communications and Networking Conference (CCNC), 2010 7th IEEE. IEEE, 1-6.

[12] Syed Faisal A Shah, Seshan Srirangarajan, and Ahmed H Tewfik. 2010. Implementation of a directional beacon-based position location algorithm in a signal processing framework. IEEE Transactions on Wireless Communications 9, 3 (2010).

[13] Kuei-Ping Shih, Chien-Min Chou, I-Hsin Liu, and Chun-Chih Li. 2010. On barrier coverage in wireless camera sensor networks. In Advanced Information Networking and Applications (AINA), 2010 24th IEEE International Conference on. IEEE, 873-879.

[14] Lei Shu, Yan Zhang, Laurence T Yang, Yu Wang, Manfred Hauswirth, and Naixue Xiong. 2010. TPGF: geographic routing in wireless multimedia sensor networks. Telecommunication Systems 44, 1-2 (2010), 79-95.

[15] Kuo-Feng Ssu, Wei-Tong Wang, Feng-Kuang Wu, and Tzu-Ting Wu. 2009. Kbarrier coverage with a directional sensing model. International fournal on Smart Sensing and Intelligent Systems 2, 1 (2009), 75-93.

[16] Dan Tao and Tin-Yu Wu. 2015. A survey on barrier coverage problem in directional sensor networks. IEEE sensors journal 15, 2 (2015), 876-885.

[17] Inc. Wikimedia Foundation. 2017. In Amenas hostage crisis. (July 2017). https: //en.wikipedia.org/wiki/In_Amenas_hostage_crisis.

[18] Li Zhang, Jian Tang, and Weiyi Zhang. 2009. Strong barrier coverage with directional sensors. In Global Telecommunications Conference, 2009. GLOBECOM 2009. IEEE. IEEE, 1-6. 